\title{
Lymphoid and myeloid lineage commitment in multipotent hematopoietic progenitors
}

\author{
Motonari Kondo ${ }^{1,2}$ \\ ${ }^{1}$ Department of Immunology, Duke University Medical Center, Durham, North Carolina, USA \\ ${ }^{2}$ Department of Immunology, Toho University School of Medicine, Tokyo, Japan
}

\section{Summary}

\begin{abstract}
Hematopoietic stem cells (HSCs) continuously replenish all classes of blood cells through a series of lineage restriction steps that results in the progressive loss of differentiation potential to other cell lineages. This review focuses on the recent advances in understanding one of the earliest differentiation steps in HSC maturation, which involves the diversification of the lymphoid and myeloid cell lineages, the two major branches of hematopoietic cells. We discuss progress in the identification and characterization of progenitor populations downstream of HSCs, which has been a key to understanding the sequential biological events that take place along the course of differentiation into a certain hematopoietic cell type. We also discuss the importance of bone marrow microenvironment in lymphoid and myeloid lineage choice.
\end{abstract}

\section{Keywords \\ hematopoiesis; lymphopoiesis; bone marrow microenvironment; cytokine; G-protein coupled receptors}

\section{Maturation of hematopoietic stem cells}

Blood cells, which are categorized in either the lymphoid or the myeloid lineage, are generated from hematopoietic stem cells (HSCs). Lymphoid lineage cells include T, B, and natural killer (NK) cells, while megakaryocytes and erythrocytes (MegE) as well as granulocytes and macrophages (GM) belong to the myeloid lineage $(1,2)$. These two lineages are separable at the progenitor level. Common lymphoid progenitors (CLPs) can differentiate into all types of lymphocytes without noticeable myeloid potential under physiological conditions (3), although some myeloid related genes might be detected in CLPs, depending on the experimental conditions (4). Similarly, common myeloid progenitors (CMPs) can give rise to all classes of myeloid cells with no or extensively low levels of B-cell potential (5). Another cell type, dendritic cells (DCs), is not clearly grouped either in lymphoid or myeloid lineage, because DC can be arise from either CLPs or CMPs $(6,7)$. By transplanting a single HSC into a lethally irradiated recipient mouse, long-term reconstitution of both the lymphoid and the myeloid compartment was achieved (8-10), demonstrating that the HSC is the common ancestor of all blood cells.

HSCs develop into progenitors that gradually lose certain lineage differentiation potential. The loss of potential to one cell lineage results in the commitment of progenitors into

Correspondence to: Motonari Kondo, Department of Immunology, Duke University Medical Center, 101 Jones Building, DUMC 3010, Research Drive, Durham, NC 27710, Tel.: +1 919613 7809, Fax: +1 919613 7852, motonari.kondo@ duke.edu or motonari.kondo@med.toho-u.ac.jp. 
alternative cell lineages (11). In response to the earliest differentiation signals, HSCs gradually lose their self-renewal ability (1). Self-renewal is the ability to proliferate while fully maintaining the differentiation potential of the parental cells, which is one of the unique features possessed by all types of stem cells. This self-renewal ability subdivides the steady state HSC pool into two populations: long-term (LT) and short-term (ST) HSCs (12-15). LT-HSCs have life-long self-renewal ability and contribute to long-term multilineage reconstitution of irradiated hosts upon transplantation. In contrast, ST-HSCs have limited self-renewal ability and can only support reconstitution of the hematopoietic system for about 6 weeks $(12,14,16)$. ST-HSCs give rise to multipotent progenitors (MPPs) (13). MPPs can support the generation of all mature blood cells types but maintain no obvious self-renewal capacity, and as a consequence, MPPs can only support hematopoiesis transiently $(12,14)$. Furthermore, it has been demonstrated that these aforementioned progenitor populations form a sequential developmental lineage where LT-HSCs $\rightarrow$ STHSCs $\rightarrow$ MPPs $(11,13)$. All of these cells are included in the c-Kit ${ }^{\text {hi }}$ Lineage ${ }^{-}$Sca- $1^{+}$(KLS) bone marrow fraction $(11,14)$. The lack of self-renewal activity along with their ability to give rise to lineage-restricted oligopotent progenitors has led to the speculation that the first step of lineage restriction occurs in MPPs during blood cell development.

\section{Diversification of lymphoid and myeloid lineages}

Subsequent studies in fetal hematopoiesis suggested that the divergence of the lymphoid and myeloid lineages might not be as simplistic as previously thought. Although the fetal counterpart of adult CMPs has been identified (17), the existence of lymphoid committed progenitors has yet to be demonstrated in fetal liver. Cells with the phenotype of adult CLPs are present in the fetal liver; however, these cells can give rise to macrophages in addition to $\mathrm{T}$ and $\mathrm{B}$ cells (18). While no T/B bipotent readout was found in fetal liver progenitors on the clonal level, progenitors with only T/B/GM, T/GM, and B/GM differentiation potential are present (19). Because of these findings, it has been speculated that clear lymphoid and myeloid lineage segregation does not occur during fetal hematopoietic differentiation. It is unclear whether the differences observed in fetal and adult hematopoiesis are due to different intrinsic mechanisms in fetal and adult progenitors or due to fetal and adult progenitors developing in different microenvironments [fetal liver vs. bone marrow (BM)]. It is also possible that the different models proposed are simply due to the inability at the present time to isolate the adult counterpart of developmental intermediates in the fetal liver and vice versa. The following sections discuss how this central question has been clarified by more recent evidence.

Various hematopoietic and lymphoid progenitors, such as MPPs and CLPs, mobilize from BM and initiate T cell development in the thymus (20). It seems that MPPs, especially $\mathrm{CCR}^{+}$MPPs, are the major population of BM cells that transit to the thymus (21). CCR9 ${ }^{+}$ MPPs are subpopulation of vascular cell adhesion molecule (VCAM-1) ${ }^{-}$MPPs, which are categorized in lymphoid primed MPPs (LMPPs) $(22,23)$ and have the potential to give rise to T, B, and GM cells $(23,24)$. Once the hematopoietic progenitors home to the thymus, Bcell development potential but not myeloid potential is immediately shut off by stimulation through Notch (25-27). This finding suggests that lymphoid and myeloid lineage segregation does not necessarily occur before loss of B-cell potential. Based on this evidence, a revised myeloid biased hematopoietic differentiation model was proposed (28).

Further characterization of the MPP population in the bone marrow has provided a higher resolution of the hematopoietic tree and a revision to the previously proposed 'classical' model. These studies have also suggested a more similar differentiation program during fetal and adult hematopoiesis. Heterogeneity of the MPP population defined as Flt3 ${ }^{+} \mathrm{KLS}$ has been implicated $(12,14)$. Indeed, the Flt $3^{+}$Thy $-1.1^{-}$KLS population (MPP) can be divided 
into the three subpopulations based on Flt3 and VCAM- 1 expression (Flt $3^{\text {lo }}$ VCAM- $1^{+}$, Flt $3^{\text {hi VCAM }}-1^{+}$, and Flt $3^{\text {hi }}$ VCAM- $\left.{ }^{-}\right)(23,24)$. Analysis of these three MPP subpopulations indicates the lymphoid and myeloid lineage branching point and a step-wise lineage restriction process toward lymphoid lineage commitment (11). The most immature Flt $3^{\text {lo }}$ VCAM- $1^{+}$MPPs have full multipotent differentiation potential, which differs from the other two MPP subpopulations. Therefore, Flt $3^{\text {lo }} \mathrm{VCAM}-1^{+}$MPPs are by definition 'classical' MPPs. The more mature Flt $3^{\text {hi }}$ VCAM- $1^{+}$MPPs can give rise to GM cells and lymphocytes as efficiently as Flt $3^{\text {lo }}$ VCAM- $1^{+}$MPPs do (23). However, Flt $3^{\text {hi }}$ VCAM- $1^{+}$MPPs have significantly lower MegE potential, both in vitro and in vivo. In addition, Flt ${ }^{\text {lo }}$ VCAM- $1^{+}$ MPPs but not Flt $3^{\text {hi }}$ VCAM- $1^{+}$MPPs can give rise to CMPs, suggesting that the Flt $3^{\text {lo }}$ VCAM $-1^{+}$MPP population is the branching point of the myeloid lineage (23). The most mature Flt $3{ }^{\text {hi }}$ VCAM $-1^{-}$MPPs have significantly lower GM and almost no MegE potential with high lymphoid potential, comparable to other MPP subfractions $(23,24)$. Although MegE potential may not be completely silenced in the Flt ${ }^{\text {hi }}$ VCAM- $1^{-}$MPP population (29), the differential potential of the three MPP subpopulations described above suggests that MPPs first lose MegE and then GM potential before establishing lymphoid lineage commitment at the CLP stage.

A more comprehensive analysis of lymphoid priming status of VCAM-1 $1^{-}$MPPs was performed recently with recombination-activating gene 1-green fluorescent protein (RAG1GFP) reporter mice (30). The results of this investigation showed that VCAM- $1^{-}$MPPs can be further subdivided into $\mathrm{RAG} 1^{-}$and $\mathrm{RAG} 1^{+}$populations (31). VCAM- $1^{-} \mathrm{RAG} 1^{+}$cells might be immediately upstream of CLPs, because myeloid potential in VCAM-1 ${ }^{-} \mathrm{RAG} 1^{+}$ cells is extremely low both in vitro cultures and in vivo (31). In contrast, VCAM- $1^{-} \mathrm{RAG} 1^{-}$ cells can form as many GM colonies as VCAM- $1^{+}$MPPs, suggesting that suppression of GM potential has been initiated at the VCAM- $1^{-} \mathrm{RAG} 1^{+}$cell stage but not at the VCAM- $1^{-} \mathrm{RAG}^{-}$cell stage. Notably, the myeloid potential of VCAM- $1^{-} \mathrm{RAG} 1^{-}$cells in vivo is as low as in VCAM- $1^{-} \mathrm{RAG} 1^{+}$cells (31). It seems therefore that lymphoid lineage specification occurs in VCAM-1 ${ }^{-} \mathrm{RAG} 1^{-}$cells under physiological conditions in vivo. An important question is why there is a discrepancy in myeloid potential of VCAM-1 ${ }^{-} \mathrm{RAG1}{ }^{-}$ cells observed in in vitro and in vivo settings. We discuss this issue in later sections.

\section{Regulation of hematopoiesis by cytokines}

Since semi-solid medium with agarose and methylcellulose have been applied to the culture of hematopoietic progenitors, extensive efforts have been put into the investigation to clarify the nature and regulatory mechanisms of lineage commitment. It was originally observed that after culture of some cell lines and primary cells in conditioned medium, colonies containing various lineage cells are formed (32). Notably, certain kinds of conditioned medium were specifically required for colony formation of a given lineage. Based on this observation, lineage-specific colony stimulating factors (CSFs) were inferred to exist. In fact, various colony stimulating factors, such as granulocyte colony stimulating factor (G$\mathrm{CSF}$ ) and macrophage colony-stimulating factor (M-CSF) were then cloned from culture supernatants (32). As predicted, specific types of colonies were obtained in the presence of recombinant CSFs or cytokines. Availability of recombinant cytokines has accelerated our understanding of the lineage commitment scheme of GM and MegE lineages $(32,33)$.

In vitro culture of lymphoid progenitors was not as easy as culturing developing myeloid cells. Whitlock and Witte (34) established conditions for BM culture, in which developing B cells can be obtained at high efficiency. A key feature of this system is to establish BM stromal cell layers, on which lymphoid progenitors can differentiate into more mature B cells. Similarly, hematopoietic progenitors in BM preferentially give rise to myeloid cells in the Dexter culture conditions (35). The reason why the two different culture conditions are 
preferable for lymphoid and myeloid cells, respectively, is thought to be that different types of BM stromal cells are established under the different conditions. In fact, established BM stromal cell lines have differing abilities to support lymphoid and myeloid cell development (36). Nishikawa and colleagues (37) found that the lack of interleukin-7 (IL-7) production is a major reason why some stromal cell lines cannot support B-cell development. Through in vivo studies primarily in knockout mice, it is now well known that IL-7 is indispensable for lymphocyte development $(38,39)$. Thus, the BM microenvironment, which is composed of stromal cells and other non-hematopoietic cells, is heterogeneous in terms of its ability to support B-cell development.

By adding IL-7 to methylcellulose medium, we can observe B-cell colonies from hematopoietic progenitors (40). Semi-solid culture allows us to examine differentiation of each progenitor at the clonal level (32). However, semi-solid medium culture (like other in vitro cultures) is not perfect. Single highly purified HSCs can give rise to all hematopoietic lineage cells in vivo. Although almost all such stem cells have in vivo reconstitution potential (10), only a portion of these stem cells can form B-cell colonies in vitro (40). This discrepancy can be explained by multiple ways. One certain fact is that multiple cytokines are necessary for survival and proliferation of HSCs and progenitors, at least in in vitro cultures. Lack of such factors may reduce the plating efficiency of HSCs and early hematopoietic progenitors. Another possibility is that some factors, which are necessary for the maintenance of multi-potent differentiation potential of HSCs in vivo, are absent in vitro. None of the existing in vitro cultures can support the maintenance of HSC activity; the selfrenewal potential of HSCs is lost during culture. It is also possible that HSCs require multiple steps to commit to the lymphoid lineage, whereas myeloid differentiation can occur immediately in HSCs and other downstream progenitors. If so, hematopoietic progenitors can give rise to myeloid cells any moment before progenitors commit to the lymphoid lineage. Some cytokines, such as c-Kit ligand [also called stem cell factor (SCF)], are necessary for maintaining HSC survival. However, at the same time, these cytokines may trigger myeloid differentiation in HSCs. SCF is known to support granulocyte colony formation of bone marrow cells in methylcellulose, although SCF mostly supports survival of HSCs but not differentiation $(41,42)$.

The importance of IL-7 in lymphocyte development has been highlighted by the phenotype of IL-7-deficient mice as well as those of mice with deletions of the genes encoding IL-7 receptor subunits, IL-7R $\alpha$ or common $\gamma$ chain $\left(\gamma_{c}\right)(43)$. Through investigating the expression of IL-7R $\alpha$ in hematopoietic progenitors, the CLP population was identified. Therefore, IL-7R $\alpha$ is thought to be a marker that indicates initiation and/or commitment of developing blood cells into the lymphoid lineage. This notion was recently revisited by using genemarking methods in mice with Cre knocked-in to the IL-7R $\alpha$ gene locus (44). The majority of the earliest T-cell precursors (ETPs) in the thymus show that they have expressed IL-7R previously, although these ETPs do not express IL-7R $\alpha$ as previously reported (45). Therefore, it seems that expression of IL-7R $\alpha$ in T-cell precursors fluctuates prior to Tlineage commitment. More importantly, in contrast to the conclusion drawn by two groups (26,27), lymphoid and myeloid lineage cells are clearly separated by this gene marking method (44), suggesting that classical lymphoid and myeloid segregation might occur in physiological conditions in vivo. Again, we must carefully interpret the results from any experimental setting. Especially in the field of developmental biology, we should clearly distinguish the differentiation potential of the progenitors and contribution of the cells to commit to certain cell lineages. Further analyses are necessary to draw true fate mapping of various hematopoietic and lymphoid progenitors. 


\section{Two different modes of cytokine actions in lineage commitment}

CSFs and other lineage-affiliated cytokines preferentially support their cognate lineage cell development in in vitro cultures. Two different modes of cytokine functions, stochastic and instructive actions, in regulation of lineage commitment have been proposed (46). Several studies with genetically modified mice implied that cytokine functions in lineage commitment (or specification) are stochastic (or supportive) rather than instructive (47-50). Although lineage-specific cytokines exist, in many cases lineage specificity is due to the lineage-specific expression of the cytokine receptors, rather than the transduction of unique differentiation signals. For example, while signaling through the thrombopoietin (TPO) receptor is indispensable for megakaryocyte differentiation, replacement of the cytoplasmic region of TPO receptor with that of the G-CSF receptor supports normal megakaryopoiesis and platelet formation (51). Similarly, swapping of the cytoplasmic domains of G-CSF receptor with that of erythropoietin (EPO) receptor does not preferentially drive erythroid differentiation at the expense of granulopoiesis in mice $(49,52)$. These results suggest that different cytokine receptors can deliver the same signals to support the maturation of multiple cell lineages.

A supportive rather than instructive role of cytokine receptor signaling is also illustrated by the studies of CSFs in myeloid cell differentiation. IL-3 (multi-CSF), GM-CSF, M-CSF, and G-CSF can potently stimulate hematopoietic progenitors to induce GM differentiation in vitro. However, animals deficient in GM-CSF do not have major perturbations in hematopoiesis (53). M-CSF and G-CSF-deficient mice have reduced numbers, but not a complete absence, of circulating monocytes and neutrophils, respectively (54). Monocytes and neutrophils are still present in the absence of all G-CSF, GM-CSF, and M-CSF (55). These results suggest that CSFs are not involved in providing lineage-specific signals in HSCs or MPPs to promote GM differentiation but rather are involved in the survival and/or expansion of progenitors in vivo at the steady state. These cytokines may be necessary for terminal maturation stages rather than the commitment stage. The role of cytokines in promoting the survival of developing hematopoietic progenitors was further demonstrated when anti-apoptotic factor Bcl-xL was overexpressed in EPO-deficient mice, which was sufficient to rescue erythroid differentiation in the absence of EPO (56). Similarly, enforced expression of Bcl-2 can relieve the blockade of T-cell maturation in mice deficient with IL-7R $\alpha$ or $\gamma_{c}$, the components of the IL-7 receptor complex $(57,58)$.

Though the role of cytokines in survival and proliferation of hematopoietic progenitors is well documented, there are also examples of the involvement of cytokines in lineage specification or differentiation events. Study of the role of IL-7 receptor signaling in B-cell development has revealed its function in the differentiation of this cell lineage. While Bcl-2 overexpression can reverse the T-cell developmental blockade in IL-7R $\alpha$-deficient mice as mentioned above, B-cell development is not rescued (58). IL-7 is also not involved in lymphoid specification or commitment, as IL-7-deficient mice do not have a decreased number of CLPs (59). Rather, it has been shown that IL-7 receptor signaling can directly regulate immunoglobulin gene rearrangement via signal transducer and activator of transcription 5 (STAT5) at the proB-cell stage, after B-cell lineage commitment (60). More recently, it has also been shown that IL-7 stimulation is important in maintaining EBF expression, a transcription factor indispensable for B-cell development, in pre-proB cells $(59,61,62)$. This is critical for maintaining B-cell differentiation potential and progression of pre-proB cells to the pro-B-cell stage (59).

There are also examples of instructive effects of cytokines in determining cell fate decisions in oligopotent hematopoietic progenitors. Stimulation by IL-2 through ectopically expressed IL-2 receptor in CLPs can convert their cell fate from lymphoid to myeloid (3). Similarly, 
GM-CSF receptor signaling cannot substitute for signals delivered by IL-7 in IL-7 receptordeficient lymphoid progenitors but rather redirects them to the myeloid cell lineage (63). More recently, G-CSF has also been shown to upregulate the level of expression of C/EBP $\alpha$, a transcription factor critical for granulopoiesis, to specify granulocyte cell fate from GM bipotent progenitors (64). Finally, Rieger et al. (65) demonstrated by using gene-marking methods that M-CSF and G-CSF can instruct common myeloid progenitors to monocytes/ macrophages and granulocytes, respectively, in in vitro cultures. Despite accumulating evidence of instructive action of cytokines in myeloid lineage choice, it remains unclear whether stimulation by extrinsic factors is involved in lymphoid vs. myeloid cell fate determination from MPPs.

\section{Bone marrow stromal cells that form bone marrow microenvironment}

$\mathrm{BM}$ is present in the medullary cavities of all animal bones. Unlike secondary lymphoid organs such as spleen with distinct gross structures including red and white pulp, BM has no clear structural features, except for the endosteum that contains osteoblasts. The endosteum region comes in contact with calcified hard bones and provides a special microenvironment to HSCs, which is necessary for the maintenance of HSC activity $(66,67)$. There is a central $\mathrm{BM}$ sinus, and some results suggest that blood cell maturation occurs from the endosteum to central sinus (68). In fact, HSCs localize at the endosteum in close proximity to osteoblasts (66). However, similar to the gross structure of BM, antibodies for markers that define various hematopoietic progenitors stain bone marrow in a scattered or homogenous fashion, suggesting that hematopoietic progenitors may not have any specific localization pattern in BM. In accordance with this notion, no specific distribution or colonization pattern of BM stromal cells is observed. It is possible, though, that lack of useful markers for BM stromal cells prevent us from visualizing a heterogeneous structure.

Although there is no distinct structure in BM, we recently divided the BM between the endosteum and central sinus into the outer and inner regions (31) (Fig. 1). We purified various hematopoietic progenitors and injected them into mice and examined where the cells localized in BM. We found that VCAM-1 ${ }^{-}$MPPs (or LMPPs) localize more proximal to the central sinus (inner region), whereas GM progenitors (GMPs), which have committed into the myeloid lineage, localize to the proximal region of endosteum (outer region). This finding does not address whether certain extrinsic factors instruct MPPs towards the myeloid lineage or simply play a supportive role, like many other myeloid growth factors $(49,51,52)$. It is possible that extracellular stimuli are necessary for proper myeloid cell development from HSCs, because the MEK/ERK pathway plays an important role in myeloid differentiation, most likely at the onset of myeloid lineage commitment during hematopoiesis (69). Since lineage conversion of CLPs from the lymphoid to myeloid lineage is possible, we hypothesize that the myeloid developmental program is dominant over the lymphoid differentiation program $(3,69)$. Therefore, we propose here that a lack of the stimulation that leads HSCs and MPPs to myeloid lineage might be a prerequisite for lymphoid lineage priming and commitment. In this sense, there must be a microenvironment in the outer region, where no active myeloid differentiation cue is present, which is similar to the inner region.

Heterogeneity of BM stromal cells has been recognized through the use of stromal cell lines that can support formation of hematopoietic co-cultures with a cobble stone appearance, or through the production of B cells from developing blood cells from fetal liver and BM $(70,71)$. Different characteristics of stromal cell lines are in part due to production of cytokines or expression of adhesion molecules. For example, as discussed above, a BM stromal cell line, PA6, cannot support B-cell development without exogenous addition of IL-7 into the culture medium (37). In contrast, another stromal line, ST2, which 
constitutively produces IL-7, can support B-cell development from mouse BM. This observation indicates that supplement of cytokines can overcome a deficiency of cytokine production by a stromal cell. OP9 is a commonly used BM stromal cell line, which is established from M-CSF-deficient OP/OP mice (72). OP9 cells are used not only for cocultures with hematopoietic cells but for initiation of blood cell development from ES cells (73). Furthermore, OP9 cells stably transfected with delta-like ligand 1 or 4 are used in in vitro cultures from hematopoietic progenitors from fetal liver and BM to support T-cell development $(74,75)$. Although OP9 cells support B-cell development from HSCs, it is not efficient enough without addition of Flt3 ligand, because Flt3 ligand is necessary for early B-cell development and OP9 cells do not produce Flt3 ligand (76). Since OP9 cells can effectively support hematopoiesis from HSCs, a search for the molecules that are involved in stromal cell-HSC interaction has been carried out. One such example is mKirre, a mammalian homolog of the gene kirre of Drosophila melanogaster, which encodes a type I membrane protein (77). If mKirre expression is suppressed by siRNA for mKirre, OP9 cells lose the potential to support hematopoiesis from HSCs. Other established BM stromal cell lines have also been used in co-culture with hematopoietic progenitors. Nonetheless, precise characterization of freshly isolated BM stromal cells has not been done extensively. To clarify the architecture of BM microenvironment, further investigation of BM stromal cells is necessary.

\section{GPCR and interaction of hematopoietic progenitors and BM stromal cells}

Guanine-nucleotide-binding proteins (G-proteins) have diverse biological functions. Gproteins are associated with seven transmembrane proteins, which are called G-proteincoupled receptors (GPCRs) (78). GPCRs are integral membrane proteins that are quite distinct from growth-factor receptors. Growth-factor receptors signal through tyrosine kinases and do not use heterotrimeric G-proteins. However, GPCRs and growth-factor receptors do share several common final pathways of signaling. Nevertheless, GPCRs, especially chemokine receptors, have distinct functions to regulate chemotactic action of cells (79). This action can determine the localization of cells at the specific sites in the organ. It is well-known that some chemokine/chemokine receptor axis plays a critical role in organization of the secondary structure of spleen and lymph nodes. Although structural features of BM are not clear except in the sternum region, chemokine receptors and/or other GPCRs may play an important role in lymphoid and myeloid lineage decision by MPPs and possibly organization of the secondary structure of BM. This is hypothesized from the fact that localization and differentiation of hematopoietic progenitors can be affected by treatment of G-protein inhibitor, pertussis toxin (PTX) (31), as mentioned below.

VCAM-1 ${ }^{-} \mathrm{RAG}^{-}$MPPs have been observed to be specific for the lymphoid lineage in vivo; however, these cells still maintain high GM potential, which is revealed in the presence of myelo-monocytic cytokines in in vitro cultures. This discrepancy is caused by GPCRmediated regulation of VCAM- $1^{-} \mathrm{RAG}^{-}$MPP localization in BM, because PTX-treated VCAM- $1^{-} \mathrm{RAG1}^{-}$MPPs regain high myeloid potential in vivo (31). More importantly, VCAM- $1^{-}$MPPs reside in the inner region of bone marrow in femurs, whereas PTX-treated VCAM- $1^{-} \mathrm{RAG1}^{-}$MPPs localize at the endosteal and outer regions, where CMPs are present (31) (Fig. 1). Therefore, it seems that GPCR-mediated signaling might play a critical role in lymphoid and myeloid lineage decision by MPPs in the physiological condition at the steady state.

Plt mice, which lack CCL19/21 production, have a disorganized structure of the secondary lymphoid organs (80), suggesting that CCL21/21 play a crucial role in the secondary structure of lymph nodes and spleens. However, no obvious difference in hematopoiesis and lymphopoiesis is observed in Plt mice. Therefore, different chemokines or ligands for 
GPCRs other than CCL19/21 should be required for the regulation of the myeloid and lymphoid lineage decision by MPPs. CXCL12 (or SDF1) is a chemokine which is necessary for retention of HSCs in BM. SDF1 also plays an important role in B lymphopoiesis, because the mice that lack SDF1 or its receptor, CXCR4, have severely reduced B-cell numbers (81). Early developing B cells, such as pre-proB cells (or CLP2) are associated with the stromal cells that produce SDF1 (82), suggesting that the SDF1/CXCR4 axis regulates localization of pre-proB cells into the specific $\mathrm{BM}$ microenvironment. More mature proB cells, however, are in contact with IL-7-producing BM stromal cells (82). Since these IL- $7^{+}$reticular cells do not express SDF1, chemokine receptors other than CXCR4 or GPCRs on proB cells are assumed to be involved in the progression of proB cells to IL-7 ${ }^{+}$ reticular cells. Further investigation is necessary to fully understand the function of GPCR in hemato/lymphopoiesis in BM.

\section{Change of microenvironment and rapid supply of myeloid cells}

Investigation of hematopoiesis and lymphopoiesis in vivo has been focused mostly on the steady state where homeostasis of blood cells is maintained. However, neutrophilia occurs rapidly upon inflammation caused by various mechanisms such as microbe infection (83). This is caused by dominant myeloid cell development with suppression of lymphocyte development and is known as emergency granulopoiesis (84). Although neutrophil counts in the blood are routinely monitored in clinics as indicators of bacterial infection, the precise mechanisms of emergency granulopoiesis have not been clarified. Components of microbes are recognized by various pattern recognition receptors, such as Toll-like receptors (TLRs). Stimulation through TLRs can direct HSCs and MPPs toward dendritic cells and result in reduction of B-cell progenitor pool (85). CSF types of cytokines also play an important role in the expansion of myeloid cells after infection, because lack of G-CSF function leads to insufficient clearance of Listeria monocytogenes (86).

Production of various cytokines, especially inflammatory cytokines, is triggered upon inflammation (87). Hematopoiesis is largely affected by these inflammatory cytokines. Among them, tumor necrosis factor $\alpha$ (TNF $\alpha$ ) seems to play a central role in the shift of BM hematopoiesis to myeloid lineage differentiation rather than lymphopoiesis. TNF $\alpha$ may directly or indirectly suppress expansion of developing B cells (88). More importantly, increase of TNF $\alpha$ inhibits SDF1 production from BM stromal cells (89), suggesting that $\mathrm{TNF} \alpha$ might change the bone marrow microenvironment. As mentioned above, SDF1/ CXCR4 is critical for B-cell development in BM. A reduced amount of SDF1 in BM cannot retain developing $\mathrm{B}$ cells in $\mathrm{BM}$, resulting in mobilization of developing $\mathrm{B}$ cells in the periphery. Empty space allows expansion of developing myeloid lineage cells. Notably, GM cell numbers are clearly increased, whereas erythroid cell numbers are not significantly different in the presence and absence of exogenous TNF $\alpha$ (90), suggesting that the same BM microenvironment can support both GM and lymphocyte development. Only minimal effects are observed in granulopoiesis in the presence of exogenous TNF $\alpha$ (90); therefore, other inflammatory cytokines must be involved in emergency granulopoiesis.

Administration of IL-1, which has been suggested to play a role in granulopoiesis, increases the granulocyte numbers but does not affect B-cell development in BM. Importantly, coinjection of TNF $\alpha$ and IL-1 recaptures the change of hematopoiesis induced by inflammation, at least adjuvant-induced inflammation (90). Therefore, the synergistic effect of TNF $\alpha$ and IL-1 might be a key to enhance granulopoiesis and inhibit B lymphopoiesis in BM upon inflammation. Since blood levels of TNFa and IL-1 are significantly increased during inflammation, the effects of these factors are systemic. As is the case of SDF1, gene expression patterns of BM stromal cells have been influenced by these inflammatory cytokines. Accordingly, more precise analyses of BM microenvironment and direct/indirect 
effects of cytokines to hematopoietic cells are necessary for complete understanding of bone marrow microenvironment.

\section{Concluding remarks}

The development of in vitro culture systems of hematopoietic and lymphoid progenitors has enabled us to examine the molecular mechanisms of cell proliferation, differentiation, and cell survival supported mainly by cytokines and adhesion molecules. The mode of cytokine functions in hematopoiesis has also been proposed and discussed extensively. However, in vitro approaches may not be ideal, because a culture system is more or less artificial. Therefore, in vivo approaches with the techniques of BM transplantation or adoptive transfer of cells are more physiological. However, after total body irradiation to make 'space' in BM before injection of hematopoietic progenitors, massive systemic inflammation is observed. Therefore, some data obtained using this experimental system may not be readily integrated into to the hematopoietic tree at the steady state, even if the data were obtained in an in vivo setting. Effects of the BM microenvironment in lymphoid and myeloid specification/ commitment during the steady state and in specialized conditions, such as inflammation, are not new concepts, but they have yet to be extensively investigated by researchers in the field of hematology and immunology. We have tried to better understand the molecular regulation of lineage commitment at the cellular and molecular levels, mainly focusing on hematopoietic progenitors. To this end, it may be time to consider the influence of extracellular factors on the lineage choice by MPPs. In addition of course, we need to clarify key transcription or nuclear factors that may play a role in specification and/or commitment to the lymphoid and myeloid lineages. Fluorescence-activated cell sorting (FACS) has become an indispensable tool for analyzing hematopoietic cell populations. However, another technical breakthrough may be necessary to provide us with new insight into this field. We believe these advances are coming soon.

\section{Acknowledgments}

I thank Anne Lai for critical reading of this manuscript. This study was funded by NIH R01 CA098129 and has been funded by NIH R01 AI056123 and Alex Lemonade Stand Foundation for Childhood Cancer. Motonari Kondo is a Scholar of the Leukemia \& Lymphoma Society.

\section{References}

1. Kondo M, et al. Biology of hematopoietic stem cells and progenitors: implications for clinical application. Annu Rev Immunol. 2003; 21:759-806. [PubMed: 12615892]

2. Weissman IL. Translating stem and progenitor cell biology to the clinic: barriers and opportunities. Science (New York, NY. 2000 Feb 25; 287(5457):1442-6.

3. Kondo M, Scherer DC, Miyamoto T, King AG, Akashi K, Sugamura K, et al. Cell-fate conversion of lymphoid-committed progenitors by instructive actions of cytokines. Nature. 2000 Sep 21; 407(6802):383-6. [PubMed: 11014194]

4. Delogu A, Schebesta A, Sun Q, Aschenbrenner K, Perlot T, Busslinger M. Gene repression by Pax5 in B cells is essential for blood cell homeostasis and is reversed in plasma cells. Immunity. 2006 Mar; 24(3):269-81. [PubMed: 16546096]

5. Akashi K, Traver D, Miyamoto T, Weissman IL. A clonogenic common myeloid progenitor that gives rise to all myeloid lineages. Nature. 2000 Mar 9; 404(6774):193-7. [PubMed: 10724173]

6. Manz MG, Traver D, Miyamoto T, Weissman IL, Akashi K. Dendritic cell potentials of early lymphoid and myeloid progenitors. Blood. 2001 Jun 1; 97(11):3333-41. [PubMed: 11369621]

7. Traver D, Akashi K, Manz M, Merad M, Miyamoto T, Engleman EG, et al. Development of CD8alpha-positive dendritic cells from a common myeloid progenitor. Science (New York, NY. 2000 Dec 15; 290(5499):2152-4. 
8. Smith LG, Weissman IL, Heimfeld S. Clonal analysis of hematopoietic stem-cell differentiation in vivo. Proceedings of the National Academy of Sciences of the United States of America. 1991; 88(7):2788-92. [PubMed: 1672767]

9. Osawa M, Hanada K, Hamada H, Nakauchi H. Long-term lymphohematopoietic reconstitution by a single CD34-low/negative hematopoietic stem cell. Science (New York, NY. 1996 Jul 12; 273(5272):242-5.

10. Matsuzaki Y, Kinjo K, Mulligan RC, Okano H. Unexpectedly efficient homing capacity of purified murine hematopoietic stem cells. Immunity. 2004 Jan; 20(1):87-93. [PubMed: 14738767]

11. Lai AY, Kondo M. T and B lymphocyte differentiation from hematopoietic stem cell. Semin Immunol. 2008 Aug; 20(4):207-12. [PubMed: 18583148]

12. Morrison SJ, Weissman IL. The long-term repopulating subset of hematopoietic stem cells is deterministic and isolatable by phenotype. Immunity. 1994; 1(8):661-73. [PubMed: 7541305]

13. Morrison SJ, Wandycz AM, Hemmati HD, Wright DE, Weissman IL. Identification of a lineage of multipotent hematopoietic progenitors. Development. 1997 May; 124(10):1929-39. [PubMed: 9169840]

14. Christensen JL, Weissman IL. Flk-2 is a marker in hematopoietic stem cell differentiation: a simple method to isolate long-term stem cells. Proceedings of the National Academy of Sciences of the United States of America. 2001 Dec 4; 98(25):14541-6. [PubMed: 11724967]

15. Kiel MJ, Yilmaz OH, Iwashita T, Terhorst C, Morrison SJ. SLAM family receptors distinguish hematopoietic stem and progenitor cells and reveal endothelial niches for stem cells. Cell. $2005 \mathrm{Jul}$ 1; 121(7):1109-21. [PubMed: 15989959]

16. Yang L, Bryder D, Adolfsson J, Nygren J, Mansson R, Sigvardsson M, et al. Identification of Lin(-)Sca1(+)kit(+)CD34(+)Flt3- short-term hematopoietic stem cells capable of rapidly reconstituting and rescuing myeloablated transplant recipients. Blood. 2005 Apr 1; 105(7):271723. [PubMed: 15572596]

17. Traver D, Miyamoto T, Christensen J, Iwasaki-Arai J, Akashi K, Weissman IL. Fetal liver myelopoiesis occurs through distinct, prospectively isolatable progenitor subsets. Blood. 2001; 98(3):627-35. [PubMed: 11468160]

18. Mebius RE, Miyamoto T, Christensen J, Domen J, Cupedo T, Weissman IL, et al. The fetal liver counterpart of adult common lymphoid progenitors gives rise to all lymphoid lineages, CD45+CD4+CD3- cells, as well as macrophages. Journal of Immunology. 2001; 166(11):6593601.

19. Kawamoto H, Ohmura K, Katsura Y. Presence of progenitors restricted to T, B, or myeloid lineage, but absence of multipotent stem cells, in the murine fetal thymus. Journal of Immunology. 1998; 161(8):3799-802.

20. Petrie HT, Kincade PW. Many roads, one destination for T cell progenitors. The Journal of experimental medicine. 2005; 202(1):11-3. [PubMed: 15983068]

21. Lai AY, Kondo M. Identification of a bone marrow precursor of the earliest thymocytes in adult mouse. Proceedings of the National Academy of Sciences of the United States of America. 2007 Apr 10; 104(15):6311-6. [PubMed: 17404232]

22. Adolfsson J, Mansson R, Buza-Vidas N, Hultquist A, Liuba K, Jensen CT, et al. Identification of Flt3+ lympho-myeloid stem cells lacking erythro-megakaryocytic potential a revised road map for adult blood lineage commitment. Cell. 2005 Apr 22; 121(2):295-306. [PubMed: 15851035]

23. Lai AY, Kondo M. Asymmetrical lymphoid and myeloid lineage commitment in multipotent hematopoietic progenitors. The Journal of experimental medicine. 2006 Aug 7; 203(8):1867-73. [PubMed: 16880261]

24. Lai AY, Lin SM, Kondo M. Heterogeneity of Flt3-expressing multipotent progenitors in mouse bone marrow. J Immunol. 2005 Oct 15; 175(8):5016-23. [PubMed: 16210604]

25. Pui JC, Allman D, Xu L, DeRocco S, Karnell FG, Bakkour S, et al. Notch1 expression in early lymphopoiesis influences B versus T lineage determination. Immunity. 1999 Sep; 11(3):299-308. [PubMed: 10514008]

26. Bell JJ, Bhandoola A. The earliest thymic progenitors for T cells possess myeloid lineage potential. Nature. 2008 Apr 10; 452(7188):764-7. [PubMed: 18401411] 
27. Wada H, Masuda K, Satoh R, Kakugawa K, Ikawa T, Katsura Y, et al. Adult T-cell progenitors retain myeloid potential. Nature. 2008 Apr 10; 452(7188):768-72. [PubMed: 18401412]

28. Kawamoto H, Katsura Y. A new paradigm for hematopoietic cell lineages: revision of the classical concept of the myeloid-lymphoid dichotomy. Trends in immunology. 2009 May; 30(5):193-200. [PubMed: 19356980]

29. Forsberg EC, Serwold T, Kogan S, Weissman IL, Passegue E. New evidence supporting megakaryocyte-erythrocyte potential of flk2/flt3+ multipotent hematopoietic progenitors. Cell. 2006 Jul 28; 126(2):415-26. [PubMed: 16873070]

30. Igarashi H, Gregory SC, Yokota T, Sakaguchi N, Kincade PW. Transcription from the RAG1 locus marks the earliest lymphocyte progenitors in bone marrow. Immunity. 2002 Aug; 17(2):117-30. [PubMed: 12196284]

31. Lai AY, Watanabe A, O'Brien T, Kondo M. Pertussis toxin-sensitive G proteins regulate lymphoid lineage specification in multipotent hematopoietic progenitors. Blood. 2009 Jun 4; 113(23):575764. [PubMed: 19363218]

32. Metcalf D. Hematopoietic cytokines. Blood. 2008 Jan 15; 111(2):485-91. [PubMed: 18182579]

33. Orkin SH. Diversification of haematopoietic stem cells to specific lineages. Nat Rev Genet. 2000 Oct; 1(1):57-64. [PubMed: 11262875]

34. Whitlock CA, Witte ON. Long-term culture of B lymphocytes and their precursors from murine bone marrow. Proceedings of the National Academy of Sciences of the United States of America. 1982 Jun; 79(11):3608-12. [PubMed: 6980414]

35. Dexter TM, Allen TD, Lajtha LG. Conditions controlling the proliferation of haemopoietic stem cells in vitro. Journal of cellular physiology. 1977 Jun; 91(3):335-44. [PubMed: 301143]

36. Wineman J, Moore K, Lemischka I, Muller-Sieburg C. Functional heterogeneity of the hematopoietic microenvironment: rare stromal elements maintain long-term repopulating stem cells. Blood. 1996 May 15; 87(10):4082-90. [PubMed: 8639765]

37. Sudo T, Ito M, Ogawa Y, Iizuka M, Kodama H, Kunisada T, et al. Interleukin 7 production and function in stromal cell-dependent B cell development. The Journal of experimental medicine. 1989 Jul 1; 170(1):333-8. [PubMed: 2787384]

38. Peschon JJ, Morrissey PJ, Grabstein KH, Ramsdell FJ, Maraskovsky E, Gliniak BC, et al. Early lymphocyte expansion is severely impaired in interleukin 7 receptor-deficient mice. The Journal of experimental medicine. 1994 Nov 1; 180(5):1955-60. [PubMed: 7964471]

39. von Freeden-Jeffry U, Vieira P, Lucian LA, McNeil T, Burdach SE, Murray R. Lymphopenia in interleukin (IL)-7 gene-deleted mice identifies IL-7 as a nonredundant cytokine. The Journal of experimental medicine. 1995 Apr 1; 181(4):1519-26. [PubMed: 7699333]

40. Kondo M, Weissman IL, Akashi K. Identification of clonogenic common lymphoid progenitors in mouse bone marrow. Cell. 1997 Nov 28; 91(5):661-72. [PubMed: 9393859]

41. Domen J, Weissman IL. Hematopoietic stem cells need two signals to prevent apoptosis; BCL-2 can provide one of these, Kitl/c-Kit signaling the other. The Journal of experimental medicine. 2000 Dec 18; 192(12):1707-18. [PubMed: 11120768]

42. Domen J, Gandy KL, Weissman IL. Systemic overexpression of BCL-2 in the hematopoietic system protects transgenic mice from the consequences of lethal irradiation. Blood. 1998; 91(7): 2272-82. [PubMed: 9516125]

43. Kondo M, Weissman IL. Function of cytokines in lymphocyte development. Curr Top Microbiol Immunol. 2000; 251:59-65. [PubMed: 11036759]

44. Schlenner SM, Madan V, Busch K, Tietz A, Laufle C, Costa C, et al. Fate mapping reveals separate origins of $\mathrm{T}$ cells and myeloid lineages in the thymus. Immunity. 2010 Mar 26; 32(3): 426-36. [PubMed: 20303297]

45. Allman D, Sambandam A, Kim S, Miller JP, Pagan A, Well D, et al. Thymopoiesis independent of common lymphoid progenitors. Nature immunology. 2003 Feb; 4(2):168-74. [PubMed: 12514733]

46. Metcalf D. Stem cells, pre-progenitor cells and lineage-committed cells: are our dogmas correct? Annals of the New York Academy of Sciences. 1999; 872:289-303. discussion -4. [PubMed: 10372131] 
47. Fairbairn LJ, Cowling GJ, Reipert BM, Dexter TM. Suppression of apoptosis allows differentiation and development of a multipotent hemopoietic cell line in the absence of added growth factors. Cell. 1993 Sep 10; 74(5):823-32. [PubMed: 7690686]

48. Socolovsky M, Lodish HF, Daley GQ. Control of hematopoietic differentiation: lack of specificity in signaling by cytokine receptors. Proceedings of the National Academy of Sciences of the United States of America. 1998 Jun 9; 95(12):6573-5. [PubMed: 9618452]

49. Semerad CL, Poursine-Laurent J, Liu F, Link DC. A role for G-CSF receptor signaling in the regulation of hematopoietic cell function but not lineage commitment or differentiation. Immunity. 1999; 11(2):153-61. [PubMed: 10485650]

50. Jacob J, Haug JS, Raptis S, Link DC. Specific signals generated by the cytoplasmic domain of the granulocyte colony-stimulating factor (G-CSF) receptor are not required for G-CSF-dependent granulocytic differentiation. Blood. 1998 Jul 15; 92(2):353-61. [PubMed: 9657731]

51. Goldsmith MA, Mikami A, You Y, Liu KD, Thomas L, Pharr P, et al. Absence of cytokine receptor-dependent specificity in red blood cell differentiation in vivo. Proceedings of the National Academy of Sciences of the United States of America. 1998 Jun 9; 95(12):7006-11. [PubMed: 9618529]

52. Stoffel R, Ziegler S, Ghilardi N, Ledermann B, de Sauvage FJ, Skoda RC. Permissive role of thrombopoietin and granulocyte colony-stimulating factor receptors in hematopoietic cell fate decisions in vivo. Proceedings of the National Academy of Sciences of the United States of America. 1999; 96(2):698-702. [PubMed: 9892696]

53. Stanley E, Lieschke GJ, Grail D, Metcalf D, Hodgson G, Gall JA, et al. Granulocyte/macrophage colony-stimulating factor-deficient mice show no major perturbation of hematopoiesis but develop a characteristic pulmonary pathology. Proceedings of the National Academy of Sciences of the United States of America. 1994 Jun 7; 91(12):5592-6. [PubMed: 8202532]

54. Lieschke GJ, Stanley E, Grail D, Hodgson G, Sinickas V, Gall JA, et al. Mice lacking both macrophage- and granulocyte-macrophage colony-stimulating factor have macrophages and coexistent osteopetrosis and severe lung disease. Blood. 1994; 84(1):27-35. [PubMed: 8018921]

55. Hibbs ML, Quilici C, Kountouri N, Seymour JF, Armes JE, Burgess AW, et al. Mice lacking three myeloid colony-stimulating factors (G-CSF, GM-CSF, and M-CSF) still produce macrophages and granulocytes and mount an inflammatory response in a sterile model of peritonitis. J Immunol. 2007 May 15; 178(10):6435-43. [PubMed: 17475873]

56. Dolznig H, Habermann B, Stangl K, Deiner EM, Moriggl R, Beug H, et al. Apoptosis protection by the Epo target $\mathrm{Bcl}-\mathrm{X}(\mathrm{L})$ allows factor-independent differentiation of primary erythroblasts. Current Biology. 2002; 12(13):1076-85. [PubMed: 12121614]

57. Kondo M, Akashi K, Domen J, Sugamura K, Weissman IL. Bcl-2 rescues T lymphopoiesis, but not B or NK cell development, in common gamma chain-deficient mice. Immunity. 1997; 7(1):15562. [PubMed: 9252128]

58. Akashi K, Kondo M, von Freeden-Jeffry U, Murray R, Weissman IL. Bcl-2 rescues T lymphopoiesis in interleukin-7 receptor-deficient mice. Cell. 1997 Jun 27; 89(7):1033-41. [PubMed: 9215626]

59. Kikuchi K, Lai AY, Hsu CL, Kondo M. IL-7 receptor signaling is necessary for stage transition in adult $\mathrm{B}$ cell development through up-regulation of EBF. The Journal of experimental medicine. 2005 Apr 18; 201(8):1197-203. [PubMed: 15837809]

60. Bertolino E, Reddy K, Medina KL, Parganas E, Ihle J, Singh H. Regulation of interleukin 7dependent immunoglobulin heavy-chain variable gene rearrangements by transcription factor STAT5. Nature immunology. 2005; 6(8):836-43. [PubMed: 16025120]

61. Dias S, Silva H Jr, Cumano A, Vieira P. Interleukin-7 is necessary to maintain the B cell potential in common lymphoid progenitors. The Journal of experimental medicine. 2005 Mar 21; 201(6): 971-9. [PubMed: 15767371]

62. Kikuchi K, Kasai H, Watanabe A, Lai AY, Kondo M. IL-7 specifies B cell fate at the common lymphoid progenitor to pre-proB transition stage by maintaining early B cell factor expression. $\mathrm{J}$ Immunol. 2008 Jul 1; 181(1):383-92. [PubMed: 18566404]

63. Iwasaki-Arai J, Iwasaki H, Miyamoto T, Watanabe S, Akashi K. Enforced granulocyte/ macrophage colony-stimulating factor signals do not support lymphopoiesis, but instruct lymphoid 
to myelomonocytic lineage conversion. The Journal of experimental medicine. $2003 ; 197(10)$ : 1311-22. [PubMed: 12756267]

64. Dahl R, Walsh JC, Lancki D, Laslo P, Iyer SR, Singh H, et al. Regulation of macrophage and neutrophil cell fates by the PU.1:C/EBPalpha ratio and granulocyte colony-stimulating factor. Nature immunology. 2003; 4(10):1029-36. Epub 2003 Sep 7. [PubMed: 12958595]

65. Rieger MA, Hoppe PS, Smejkal BM, Eitelhuber AC, Schroeder T. Hematopoietic cytokines can instruct lineage choice. Science (New York, NY. 2009 Jul 10; 325(5937):217-8.

66. Wilson A, Trumpp A. Bone-marrow haematopoietic-stem-cell niches. Nature reviews. 2006 Feb; 6(2):93-106.

67. Nilsson SK, Johnston HM, Coverdale JA. Spatial localization of transplanted hemopoietic stem cells: inferences for the localization of stem cell niches. Blood. 2001 Apr 15; 97(8):2293-9. [PubMed: 11290590]

68. Nagasawa T. Microenvironmental niches in the bone marrow required for B-cell development. Nature reviews. 2006 Feb; 6(2):107-16.

69. Hsu CL, Kikuchi K, Kondo M. Activation of MEK/ERK signaling pathway is involved in the myeloid lineage commitment. Blood. 2007 May 29.

70. Charbord P, Moore K. Gene expression in stem cell-supporting stromal cell lines. Annals of the New York Academy of Sciences. 2005 Jun.1044:159-67. [PubMed: 15958709]

71. Badillo AT, Flake AW. The regulatory role of stromal microenvironments in fetal hematopoietic ontogeny. Stem cell reviews. 2006; 2(3):241-6. [PubMed: 17625260]

72. Kodama H, Nose M, Niida S, Nishikawa S. Involvement of the c-kit receptor in the adhesion of hematopoietic stem cells to stromal cells. Experimental hematology. 1994 Sep; 22(10):979-84. [PubMed: 7522185]

73. Nakano T, Kodama H, Honjo T. Generation of lymphohematopoietic cells from embryonic stem cells in culture. Science (New York, NY. 1994 Aug 19; 265(5175):1098-101.

74. Mohtashami M, Shah DK, Nakase H, Kianizad K, Petrie HT, Zuniga-Pflucker JC. Direct comparison of Dll1- and Dll4-mediated Notch activation levels shows differential lymphomyeloid lineage commitment outcomes. J Immunol. Jul 15; 185(2):867-76. [PubMed: 20548034]

75. Schmitt TM, Zuniga-Pflucker JC. Induction of T cell development from hematopoietic progenitor cells by delta-like-1 in vitro. Immunity. 2002 Dec; 17(6):749-56. [PubMed: 12479821]

76. Cho SK, Webber TD, Carlyle JR, Nakano T, Lewis SM, Zuniga-Pflucker JC. Functional characterization of B lymphocytes generated in vitro from embryonic stem cells. Proceedings of the National Academy of Sciences of the United States of America. 1999 Aug 17; 96(17):9797802. [PubMed: 10449774]

77. Ueno H, Sakita-Ishikawa M, Morikawa Y, Nakano T, Kitamura T, Saito M. A stromal cell-derived membrane protein that supports hematopoietic stem cells. Nature immunology. 2003 May; 4(5): 457-63. [PubMed: 12665856]

78. Klabunde T, Hessler G. Drug design strategies for targeting G-protein-coupled receptors. Chembiochem. 2002 Oct 4; 3(10):928-44. [PubMed: 12362358]

79. Allen SJ, Crown SE, Handel TM. Chemokine: receptor structure, interactions, and antagonism. Annual review of immunology. 2007; 25:787-820.

80. Nakano H, Mori S, Yonekawa H, Nariuchi H, Matsuzawa A, Kakiuchi T. A novel mutant gene involved in T-lymphocyte-specific homing into peripheral lymphoid organs on mouse chromosome 4. Blood. 1998 Apr 15; 91(8):2886-95. [PubMed: 9531599]

81. Nagasawa T, Hirota S, Tachibana K, Takakura N, Nishikawa S, Kitamura Y, et al. Defects of Bcell lymphopoiesis and bone-marrow myelopoiesis in mice lacking the CXC chemokine PBSF/ SDF-1. Nature. 1996 Aug 15; 382(6592):635-8. [PubMed: 8757135]

82. Tokoyoda K, Egawa T, Sugiyama T, Choi BI, Nagasawa T. Cellular niches controlling B lymphocyte behavior within bone marrow during development. Immunity. 2004 Jun; 20(6):70718. [PubMed: 15189736]

83. Quie PG. The phagocytic system in host defense. Scandinavian journal of infectious diseases. 1980 24:30-2. [PubMed: 6259718]

84. Burg ND, Pillinger MH. The neutrophil: function and regulation in innate and humoral immunity. Clinical immunology (Orlando, Fla. 2001; 99(1):7-17. 
85. Nagai Y, Garrett KP, Ohta S, Bahrun U, Kouro T, Akira S, et al. Toll-like receptors on hematopoietic progenitor cells stimulate innate immune system replenishment. Immunity. 2006 Jun; 24(6):801-12. [PubMed: 16782035]

86. Lieschke GJ, Grail D, Hodgson G, Metcalf D, Stanley E, Cheers C, et al. Mice lacking granulocyte colony-stimulating factor have chronic neutropenia, granulocyte and macrophage progenitor cell deficiency, and impaired neutrophil mobilization. Blood. 1994 Sep 15; 84(6):1737-46. [PubMed: 7521686]

87. Lin WW, Karin M. A cytokine-mediated link between innate immunity, inflammation, and cancer. The Journal of clinical investigation. 2007 May; 117(5):1175-83. [PubMed: 17476347]

88. Sedger LM, Hou S, Osvath SR, Glaccum MB, Peschon JJ, van Rooijen N, et al. Bone marrow B cell apoptosis during in vivo influenza virus infection requires TNF-alpha and lymphotoxin-alpha. J Immunol. 2002 Dec 1; 169(11):6193-201. [PubMed: 12444124]

89. Ueda Y, Yang K, Foster SJ, Kondo M, Kelsoe G. Inflammation controls B lymphopoiesis by regulating chemokine CXCL12 expression. The Journal of experimental medicine. 2004 Jan 5; 199(1):47-58. [PubMed: 14707114]

90. Ueda Y, Kondo M, Kelsoe G. Inflammation and the reciprocal production of granulocytes and lymphocytes in bone marrow. The Journal of experimental medicine. 2005 Jun 6; 201(11):177180. [PubMed: 15939792] 


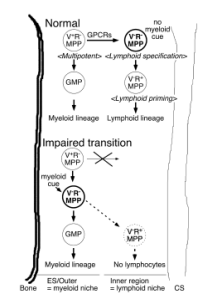

Fig. 1. Hypothetical structure of bone marrow in femurs This figure is based on the gross structure of bone marrow described in Nilsson et al. (67) and experimental results shown in Lai et al. (31). PTX-sensitive GPCR-dependent movement of MPPs from the outer to inner region is necessary for proper lymphocyte development. 